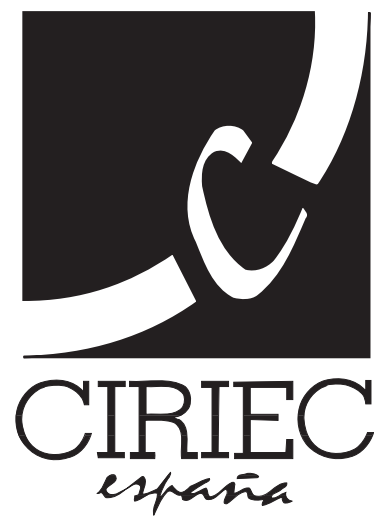

CIRIEC-España, Revista de Economía Pública, Social y Cooperativa, nº 88, Diciembre 2016, pp. 93-125

\title{
Aspectos determinantes del fracaso empresarialk efecto de la proyección social de las sociedades cooperativas frente a otras formas jurídicas
}

\author{
Cristina I. Masa Lorenzo \\ Javier Iturrioz del Campo \\ Universidad CEU San Pablo \\ Sonia Martín López \\ Universidad Complutense de Madrid
}

Cómo citar este artículo / How to cite this article: MASA, C.I., ITURRIOZ, J. \& MARTíN, S. (2016): "Aspectos determinantes del fracaso empresarial: efecto de la proyección social de las sociedades cooperativas frente a otras formas jurídicas", CIRIEC-España, Revista de Economía Pública, Social y Cooperativa, 88, 93-125.

CIRIEC-España, revista de economía pública, social y cooperativa ISSN edición impresa: 0213-8093. ISSN edición online: 1989-6816.

\section{(C) 2016 CIRIEC-España}




\title{
Aspectos determinantes del fracaso empresarial: efecto de la proyección social de las sociedades cooperativas frente a otras formas jurídicas
}

\author{
Cristina I. Masa Lorenzo \\ Javier Iturrioz del Campo \\ Sonia Martín López
}

\section{RESUMEN}

Los modelos de predicción de la insolvencia empresarial se han fundamentado en el comportamiento de un conjunto de variables económico-financieras, y otras relativas a las características de la empresa, centrados en grandes empresas. Este trabajo analiza la aplicación de un modelo a empresas de diferente tamaño, estudiando de forma especial el comportamiento de las sociedades cooperativas. Para ello, se analiza la capacidad predictiva del modelo sobre el posible fracaso empresarial, constituyéndose la solvencia, la liquidez y la rentabilidad como las principales variables para anticipar esta situación. Por otra parte, se incluye el posible efecto moderador de la forma jurídica de sociedad cooperativa en ambas variables, para determinar si las particularidades derivadas de su proyección social suponen un efecto diferenciador.

PALABRAS CLAVE: Concurso de acreedores, sociedades cooperativas, insolvencia, información económico-financiera, Modelo PLS.

CLAVES ECONLIT: M41, P13, K29.

Cómo citar este artículo / How to cite this article: MASA, C.I., ITURRIOZ, J. \& MARTÍN, S. (2016):

"Aspectos determinantes del fracaso empresarial: efecto de la proyección social de las sociedades cooperativas frente a otras formas jurídicas", CIRIEC-España, Revista de Economía Pública, Social y Cooperativa, 88, 93-125.

Correspondencia: Cristina I. Masa Lorenzo, Profesora Colaboradora Doctora, Universidad CEU San

Pablo; Javier Iturrioz del Campo, Profesor Agregado, Universidad CEU San Pablo, y Sonia Martín López, Profesor Contratado Doctor, Universidad Complutense de Madrid. E-mail de contacto:

cmasalor@ceu.es. 


\section{EXPANDED ABSTRACT}

\section{Determinants of Corporate Failure: Effect of Social Projection of Cooperative Societies against Other Legal Forms}

\section{A) Objectives}

We aim to analyze the incidence of a set of variables in business failure, analyzing whether they act in a similar way in conventional capitalist firms and cooperative societies. Specifically, a set of economic and financial variables (profitability, solvency, liquidity, turnover, economic structure, financial structure and activity) and a group of non-financial variables (size, age, industry and region), some of them considered as control variables in previous studies. In addition, the predictive capacity of the model is analyzed in order to identify the variables that allow to anticipate insolvency situations, taking as a reference the economic-financial data of the previous year of entry to the insolvency proceedings. The aforementioned study is carried out for all the companies in the sample, analyzing if there are particularities for the case of cooperative societies. For this, the moderating effect of the cooperative legal form is included, distinguishing between two subsamples of insolvent companies: one formed by cooperative societies and a second one that includes conventional capitalist firms.

\section{B) Methodology}

A review of the literature on business failure has been made, as well as on the particularities of cooperative societies, especially in economic and financial aspects and their influence on insolvency proceedings. In order to identify the main contributions to the literature and to contextualize the study object, we have used the Web of Science (WOS) and SCOPUS, edited by Elsevier, as well as other databases such as, Dialnet, Academic Search Complete, EconLit, Business Source Complete (EBSCO) and JSTOR-Business. This review allowed us to formulate several hypotheses grouped into three blocks:

- Those related to the Relationship among Profitability, Solvency and Liquidity.

- Those linked to Non-Financial Variables (characteristics of the company).

- Those related to the effect of Other Economic-Financial Ratios on Liquidity.

Taking into account these hypotheses, we formulate a model that analyzes the behavior of the companies before the business failure, identifying the same with the condition of insolvent company, being the period analyzed the included between the years 2005 and 2014, both inclusive. The data of the year prior to the entry into bankruptcy proceedings have been taken, including cooperative socie- 
ties as well as other legal forms. The model uses both financial and non-financial variables, collecting indicators of aspects such as structure or rotation, along with parameters such as age or region.

A Structural Equation Model is applied, based on Partial Least Squares, differentiating between external and internal models and statistical significance.

\section{C) Results}

The model has been evaluated according to the reliability criteria (absence of error, showing the accuracy of the measurement instrument), and validity (degree to which an indicator measures what it must measure), at two levels (construct and associated indicators) doing differently according to the relationship were Reflective (Size, Profitability, Activity, Rotation, Economic and Financial Structures), or Formative (Industry, Region and Age).

It also examines whether there is a moderating effect of the legal form on the liquidity and solvency of the companies analyzed. For this, a Multi-Group Analysis has been performed, applying the NonParametric Approach or PLS MGA. The legal form has been codified as a dichotomous dummy variable, presenting value 1 for cooperative societies and value 0 for non-cooperative societies.

Regarding the study of the significance of the hypotheses raised in the model, a procedure of bootstrapping has been implemented for all the companies involved, regardless of their legal form, and for the two differentiated groups (cooperative societies and non-cooperative societies), separating the results related to the hypotheses of the relations between profitability, solvency and liquidity, the hypotheses linked to the control variables and, finally, the hypotheses related to the effect on the liquidity of the other economic-financial variables.

From the contrast of the hypotheses put forward in the model for the totality of insolvent companies, the profitability has a positive and significant influence on the Solvency, as well as the Solvency on Liquidity, evidencing a great correlation between the solvency indicators in the short term. On the other hand, the selected Economic Structure indicators would have a small negative effect on Liquidity. Within the non-financial variables are only significant, with a lower level, on the one hand the Age on Solvency and on the other the Industry on Liquidity.

If the contrast is performed for each of the subsamples, the results show some differences of the cooperative societies in the hypotheses related to the effects of the non-financial variables on the characteristics for company on Solvency and Liquidity. However, not all of these differences are significant. It is possible to affirm that the legal form moderates only the influence of the effects of the Region and the Age on the Solvency, with a level of significance of 10 percent and 5 percent, respectively, not finding differences in the other variables analyzed. 


\section{D) Research limitations}

We can highlighted the followings limitations:

The lack of a general theory about the behavior of a company that fail. Although it is usual using economic-financial ratios model, the selection of variables is conditioned and specific for the sample on which the research is carried out, so that the predictive capacity of a model is determined by the characteristics of the sample used. Therefore, there are already studies that include other qualitative variables that can increase the predictive capacity of the models.

The absence of a consensus on the concept of firm failure, taking as reference in this research the legal criterion, being the most used in most studies, given that it has less arbitrariness. This fact implies a reduction of the size of the sample and, therefore, of the results achieved.

The difficulties and limitations of the access to the data of the companies of small dimension, characteristic that underlies in the entities object of this study.

The effect of the change in Spanish accounting regulations that occurred in 2008 in the information of the sample companies, as a consequence of the adoption of the International Accounting Standards by the European Union. It should be taken into account that the time horizon contemplated for the sample data used is the period from 2005 to 2014.

\section{E) Implications, conclusions and original value}

In view of the results obtained, Profitability, Solvency and Economic Structure anticipate possible situations of business failure. The first two have a positive effect on long-term and short-term solvency, corroborating the hypotheses raised in the bibliography consulted, while the second one would have a negative effect, evidencing that a higher result in these indicators implies a lower weight of current assets, reached lower liquidity.

When analyzing the application of the model to cooperative societies, it is verified that the three variables mentioned are valid and significant for this legal form, so the model is also applicable to them. However, there are four differentiating factors: Region, Age, Size and Financial Structure, presenting significant differences only in the first two.

KEYWORDS: Contest creditors, cooperatives, insolvency, economic information financial, Model PLS. 


\section{1.- Introducción: objetivos y metodología}

Las causas que llevan a una empresa a situaciones de fracaso empresarial pueden tener su origen en diversos motivos (Lemmon et al., 2009). A pesar del elevado volumen de investigaciones no se ha conseguido formular una teoría general sobre el fracaso empresarial, siendo muy diversas las causas que lo determinan (Manzaneque et al., 2010). Asimismo, la mayoría de estos estudios se han centrado en sociedades capitalistas, sin tener presente la problemática de otras formas jurídicas como las sociedades cooperativas ${ }^{1}$.

Las sociedades cooperativas presentan una serie de peculiaridades que les otorgan una proyección social derivada del papel que desempeñan los diferentes colectivos que las integran. Así, algunos estudios determinan que las empresas de Economía Social son las que han tenido un papel más activo en el mantenimiento de puestos de trabajo en periodos de crisis (Chaves y Monzón, 2007). Este papel se encuentra determinado por el rol que realizan los diferentes colectivos que participan en la actividad empresarial. Mientras que en una empresa convencional los proveedores, los clientes y los trabajadores de la misma no participan en la toma de decisiones, en las sociedades cooperativas estos colectivos juegan el doble papel de ser a la vez socios de la empresa. Este doble papel permite una participación de los socios en los tres tipos de flujos empresariales: productivos, económico-financieros y decisionales (Iturrioz, 2010):

- La participación de los socios en los flujos productivos se realiza de manera diferente en función del tipo de sociedad cooperativa: en las cooperativas de proveedores son los socios los que aportan sus productos a la sociedad cooperativa, en las de clientes los que adquieren dichos productos, mientras que en las de trabajadores los que aportan su trabajo.

- La participación en los flujos económico-financieros se concreta en las aportaciones realizadas al capital social, así vía remuneración de su actividad productiva: vía precios (proveedores y clientes), o salarios (trabajadores).

- Por último, la participación de los socios en la toma de decisiones se produce por medio del voto: con la peculiaridad de que cada socio tiene un voto, independientemente de su aportación al capital social, garantizándose que la toma de decisiones siempre se encuentra en manos de los socios que participan en los restantes flujos.

1.- Algunos estudios analizan el efecto de la crisis sobre las cooperativas en determinados sectores, como el caso del sector bancario realizado por Fajardo, G. (2011): "How viable are Spanish Credit Cooperatives after recent bank capitalization and restructuring regulations?", CIRIEC-España, Revista de Economía Pública, Social y Cooperativa, 73, 151-170. 
Así, mientras que en la mayoría de las formas jurídicas la toma de decisiones está en manos de socios que solo participan en la empresa mediante aportaciones financieras, en las sociedades cooperativas la toma de decisiones corresponde a socios que también ponen en juego su papel como clientes, proveedores o trabajadores de la empresa. De esta manera, decisiones de tipo económicofinanciero, que habitualmente se toman desde el punto de vista del socio capitalista, en las sociedades cooperativas toman una dimensión especial al estar ligadas a la continuidad de la empresa, con el consecuente efecto desde el punto de vista social (Iturrioz e Isabel, 2010). Esto permite una mayor flexibilidad interna para realizar ajustes y adaptarse a las condiciones del entorno, ya que al considerar la empresa como propia tienen una mayor disponibilidad para aceptar cambios en búsqueda de una estabilidad en su actividad productiva (Sáez y González, 2004, Clemente et al., 2012). En esta línea pueden destacarse estudios que ponen de manifiesto la menor sensibilidad del empleo en las sociedades cooperativas a los ciclos económicos (Calderón y Calderón, 2012, Sala et al., 2014), buscando las diferencias en aspectos relacionados con la duración de la jornada laboral o la retribución.

Estas peculiaridades han llevado a plantear como objetivo de este trabajo estudiar la incidencia en el fracaso empresarial de una serie de variables, analizando si actúan de manera similar en las empresas capitalistas tradicionales y en las sociedades cooperativas. En concreto se incluyen un conjunto de variables económicas y financieras (la rentabilidad, la solvencia, la liquidez, la rotación, la estructura económica, la estructura financiera y la actividad), y un grupo de variables no financieras (el tamaño, la antigüedad, el sector y la localización), algunas de ellas consideradas como variables de control en estudios anteriores. Además se analiza la capacidad predictiva del modelo buscando identificar las variables que permitan anticipar situaciones de insolvencia, tomando como referencia los datos económico-financieros del año previo de entrada al proceso concursal. El citado estudio se realiza para el conjunto de las empresas de la muestra, analizando si existen particularidades para el caso de las sociedades cooperativas. Para ello, se incluye el efecto moderador de la forma jurídica cooperativa, distinguiendo dos submuestras de empresas concursadas: una formada por 133 sociedades cooperativas y una segunda en la que se incluyen otras 133 empresas capitalistas convencionales.

En la metodología utilizada se diferencian cinco partes:

- Marco teórico: Se ha realizado una revisión de la bibliografía sobre el fracaso empresarial, así como sobre las particularidades de las sociedades cooperativas, especialmente en los aspectos económico-financieros y su influencia en los procesos concursales (Iturrioz, 2013). Para identificar los principales artículos y contextualizar el objeto de estudio, se ha utilizado una búsqueda en la Web of Science (WOS) y en SCOPUS editada por Elsevier, así como en otras bases de datos como, Dialnet, Academic Search Complete, EconLit y Business Source Complete (EBSCO) y JSTOR-Business. La citada revisión ha permitido formular las diferentes hipótesis que se plantean en el estudio. 
- Propuesta de Modelo: El modelo propuesto analiza el comportamiento de las empresas ante el fracaso empresarial, identificando el mismo con la condición de empresa concursada, siendo el periodo analizado el comprendido entre los años 2005 y 2014, ambos inclusive. Se han tomado los datos del año previo a la entrada en concurso, incluyendo tanto sociedades cooperativas como otras formas jurídicas.

- Selección de las variables: Para el modelo se utilizan tanto variables financieras como no financieras, recogiendo indicadores de aspectos como la estructura o la rotación, junto con parámetros como la antigüedad o la localización.

- Evaluación del Modelo: Se aplica un Modelo de Ecuaciones Estructurales, basado en Mínimos Cuadrados Parciales, diferenciando entre los modelos externo e interno y la significatividad estadística.

- Conclusiones: Por último se exponen las principales conclusiones.

\section{2.- Hipótesis planteadas}

Con la crisis económica se ha incrementado el número de entidades que buscan una solución mediante el concurso de acreedores, para intentar superar situaciones de insolvencia antes de llegar al estado de liquidación y cierre. El volumen de investigaciones orientadas a la elaboración de modelos cuantitativos y cualitativos que anticipen situaciones de fracaso empresarial ha estado unido a la etapa del ciclo económico (Pozuelo et al., 2012). Sin embargo, tras la revisión bibliográfica realizada, se observa que hasta el año 2000 la mayor parte de los estudios realizados se centran en sociedades capitalistas de gran dimensión (Altman, 1968, Altman et al., 1977, Beaver, 1966, Casey y Bartczak, 1985, Deakin, 1972, Ferrando y Blanco, 1998, Fitzpatrick, 1932, Gabás, 1990, Gentry et al., 1985 y 1987, Lizarraga, 1997, Martin, 1977, Ohlson, 1980, Platt y Platt, 1991, Sinkey, 1975, Taffler, 1983, Winakor y Smith, 1935). No obstante, en los últimos años sí se han realizado estudios que abordan la predicción del fracaso empresarial en empresas de diferente tamaño, siendo muy escasas las investigaciones sobre la problemática de algunas formas jurídicas como las sociedades cooperativas (Alfaro et al., 2008, Aguiar, 2014, Calvo-Flores et al., 2006, Correa et al., 2003, Diéguez et al., 2006, Dietrich et al., 2005, Galindo, 2006, Gómez et al., 2008, Iturrioz y Martín, 2013, Labatut et al., 2009, Manzaneque et al., 2010, Mínguez, 2006, Pozuelo et al., 2013, Pozuelo et al., 2012, Premachandra et al., 2009, Rubio, 2008). 
Teniendo en cuenta el objetivo planteado, se procede a la formulación de las hipótesis concretas que se pretenden contrastar en este estudio. Las hipótesis se han agrupado en tres bloques:

- Las relativas a la Relación entre Rentabilidad, Solvencia y Liquidez.

- Las vinculadas a las Variables No Financieras (características de la empresa).

- Las vinculadas al efecto de Otros Ratios Económico-Financieros sobre la Liquidez.

\section{A) Hipótesis relativas a la Relación entre Rentabilidad, Solvencia y Liquidez}

Son numerosos los estudios que constatan que un aumento de la rentabilidad conduce a un menor fracaso empresarial (Altman, 1968, Correa et al., 2003, Deakin, 1972, Ferrando y Blanco, 1998, Galindo, 2006, Lincoln, 1984, López et al., 1998, Ohlson, 1980). Asimismo, la rentabilidad de la empresa se configura como uno de los factores más importantes a la hora de predecir la posible situación de insolvencia de una empresa, sobre todo la rentabilidad relacionada con la inversión financiada con fondos propios, con las ventas obtenidas, así como con la deficiencia en la generación de cashflow, que las obliga a endeudarse excesivamente (Labatut et al., 2009). Por otro lado, debe tenerse en cuenta que los socios de una sociedad cooperativa no suelen evaluar su rentabilidad por su excedente contable, ni siguen una política de maximización de sus beneficios, sino que la evalúan por el diferencial de precios que la sociedad cooperativa paga a sus socios sobre el precio de mercado normal (Domingo, 2001, Gómez-Limón et al., 2003).

$H_{1}$ : la rentabilidad influye positiva y significativamente sobre la solvencia

A su vez, en la mayoría de los trabajos sobre empresas con dificultades financieras, los indicadores de mayor relevancia son los de solvencia a largo plazo y a corto plazo (Correa et al., 2003, Mateos, 2008). Cuanto mayor es la tesorería en relación con el endeudamiento, menor es la probabilidad de quiebra legal (Román et al., 2001; Lizarraga, 1997; López et al., 1998).

$\mathrm{H}_{2}$ : la solvencia influye positiva y significativamente sobre la liquidez

\section{B) Hipótesis vinculadas a Variables No Financieras (características de la empresa)}

Se incluye un conjunto de variables relativas a las características de las empresas, con el objetivo de comprobar el posible efecto negativo o positivo de cada una de ellas sobre la liquidez y sobre la solvencia de las entidades. En la revisión bibliográfica realizada se pone de manifiesto que los estudios no analizan el posible efecto de estas variables sobre los ratios de rentabilidad, por lo que se decide no estudiar dicho efecto. Estas variables son: 
Localización: dado que el estudio se centra en sociedades concursadas de España, se ha decidido analizar si la localización geográfica dentro del territorio nacional puede tener algún tipo de incidencia en la solvencia de las empresas objeto de estudio, no encontrándose la incidencia de esta variable en los estudios consultados.

Tamaño: hay autores que consideran que a mayor tamaño, menor será el riesgo esperado y menor probabilidad de fracaso empresarial (López et al., 1998). Otros destacan que los factores que afectan significativamente a la salud financiera de las empresas son el tamaño, seguido por el exceso de crecimiento y la liquidez (Chen y Wong, 2004). Sin embargo, otros establecen que no es demostrable una relación directa, creciente o decreciente, entre la solvencia y la liquidez con la dimensión empresarial, pero que se pueden obtener resultados distintos para otras definiciones de tamaño y de rentabilidad (Galindo, 2006). Además, es posible que se presenten desventajas de tipo financiero asociadas al menor tamaño de la empresa, que iría asociado al riesgo de fracaso, tales como restricciones financieras, la dificultad de acceso a la financiación a medio y largo plazo y mayor coste financiero (Hellman y Stiglitz, 2000, Maroto, 1994, Martín y Sáez, 2001, Melle, 2001).

Sector de Actividad: al igual que en la variable Tamaño, hay autores que indican que el pertenecer a un sector de actividad u otro no afecta a la insolvencia empresarial (López et al., 1998), pero, por otro lado, otros consideran que la rentabilidad y la solvencia de la empresa es claramente dependiente del sector en la clasificación tradicional de actividades, siendo directamente proporcional a la intensidad en la utilización del factor trabajo (Galindo, 2006).

Antigüedad: la literatura económica indica que el fracaso empresarial está inversamente relacionado con la edad de la empresa (Dunne et al., 1988, Mata y Portugal, 1994, Philips y Kirchhoff, 1989). No obstante, se distinguen distintos enfoques sobre cómo la edad afecta al fracaso (Henderson, 1999): el enfoque basado en el liability of newness, que postula mayores tasas de fracaso en los años iniciales porque estas tienen que utilizar recursos en aspectos tales como formar al personal o invertir en su marca (Carroll, 1983, Jovanovic, 1982), el enfoque de liability of adolescence, que señala que las tasas de fracaso más altas se obtienen unos años después de la fundación, disminuyendo con posterioridad (Bruderl y Schussler, 1990), y el enfoque liability of obsolescence, donde se pone de manifiesto que las tasas de fracaso se espera que aumenten con la edad (Barron, West y Hannan, 1994).

$H_{3}$ : la localización puede tener una influencia tanto positiva como negativa sobre la solvencia.

$H_{4}$ : la localización puede tener una influencia tanto positiva como negativa sobre la liquidez.

$H_{5}$ : el tamaño puede tener una influencia tanto positiva como negativa sobre la solvencia.

$\mathrm{H}_{6}$ : el tamaño puede tener una influencia tanto positiva como negativa sobre la liquidez.

$H_{7}$ : el sector puede tener una influencia tanto positiva como negativa sobre la solvencia.

$H_{8}$ : el sector puede tener una influencia tanto positiva como negativa sobre la liquidez.

$H_{g}$ : la antigüedad puede tener una influencia tanto positiva como negativa sobre la solvencia.

$\mathrm{H}_{10}$ : la antigüedad puede tener una influencia tanto positiva como negativa sobre la liquidez. 


\section{C) Hipótesis vinculadas al efecto de Otros Ratios Económico-Financieros sobre la Liquidez}

El tercer grupo de hipótesis refleja la posible relación de diferentes ratios con respecto a la liquidez. En concreto estas variables son:

Rotación: los ratios de rotación, vinculados con las operaciones de la actividad principal de la empresa están presentes en la mayoría de los estudios relativos a la solvencia empresarial, no siendo significativos en la mayoría de ellos. No obstante, se decide su inclusión en el modelo dada su habitualidad en la literatura sobre el fracaso empresarial (Correa et al., 2003, Diéguez, et al., 2006, Dietrich et al., 2005, Labatut et al., 2009, Mínguez, 2006; Pozuelo et al., 2012, Rubio, 2008).

Estructura Económica: Ios indicadores de estructura económica muestran una mayor capacidad para anticipar situaciones de fracaso empresarial, sobre todo si la información de las empresas se refiere a los años previos a la entrada en crisis, entendida ésta como quiebra técnica (Correa et al., 2003).

Estructura Financiera: una de las variables que contribuyen de manera más significativa en las situaciones de insolvencia son los indicadores de endeudamiento o dependencia de financiación ajena (Mateos, 2008). Varios estudios muestran que cuanto mayor es el endeudamiento de la empresa, mayor es la probabilidad de fracaso (Altman et al., 1977; Ohlson, 1980).

Actividad: los ratios relativos a la actividad están presentes en todos los estudios que versan sobre solvencia, resultando relevantes para estimar la probabilidad de que una empresa sea fracasada (Correa et al., 2003, Diéguez et al., 2006, Dietrich et al., 2005, Marí et al., 2014, Pozuelo et al., 2012).

$H_{11}$ : la rotación puede tener una influencia tanto positiva como negativa sobre la liquidez.

$\mathrm{H}_{12}$ : la estructura económica puede tener una influencia tanto positiva como negativa sobre la liquidez.

$H_{13}$ : la estructura financiera puede tener una influencia tanto positiva como negativa sobre la liquidez.

$H_{14}$ : la actividad puede tener una influencia tanto positiva como negativa sobre la liquidez. 


\section{Tabla 1. Resumen de las hipótesis del modelo propuesto}

\begin{tabular}{|l|c|l|}
\hline HIPÓTESIS & & RELACIÓN \\
\hline Rentabilidad, & $\mathrm{H}_{1}$ & La rentabilidad influye positiva y significativamente sobre la solvencia. \\
Solvencia y Liquidez & $\mathrm{H}_{2}$ & La solvencia influye positiva y significativamente sobre la liquidez. \\
\hline Variables $\mathrm{No}$ & $\mathrm{H}_{3} \mathrm{y} \mathrm{H}_{4}$ & La localización puede tener una influencia tanto positiva como negativa sobre la solvencia, y sobre la liquidez. \\
Financieras & $\mathrm{H}_{5} \mathrm{y} \mathrm{H}_{6}$ & El tamaño puede tener una influencia tanto positiva como negativa sobre la solvencia, y sobre la liquidez. \\
& $\mathrm{H}_{7} \mathrm{y} \mathrm{H}_{8}$ & El sector puede tener una influencia tanto positiva como negativa sobre la solvencia, y sobre la liquidez. \\
& $\mathrm{H}_{9} \mathrm{y} \mathrm{H}_{10}$ & La antigüedad puede tener una influencia tanto positiva como negativa sobre la solvencia, y sobre la liquidez. \\
\hline Otros Ratios & $\mathrm{H}_{11}$ & La rotación puede tener una influencia tanto positiva como negativa sobre la liquidez \\
sobre Liquidez & $\mathrm{H}_{12}$ & La estructura económica puede tener una influencia tanto positiva como negativa sobre la liquidez. \\
& $\mathrm{H}_{13}$ & La estructura financiera puede tener una influencia tanto positiva como negativa sobre la liquidez. \\
& $\mathrm{H}_{14}$ & La actividad puede tener una influencia tanto positiva como negativa sobre la liquidez. \\
\hline
\end{tabular}

FUENTE: Elaboración propia.

Con la finalidad de concretar e identificar las variables más representativas en el fracaso empresarial en las sociedades cooperativas, se han replicado las anteriores hipótesis, distinguiendo entre el comportamiento de estas frente a las sociedades no cooperativas (ver Tabla 2).

\section{Tabla 2. Resumen de las hipótesis relativas al efecto moderador de la forma jurídica del modelo propuesto}

\begin{tabular}{|c|c|c|}
\hline HIPÓ & & \\
\hline \begin{tabular}{|l|} 
Rentabilidad, \\
Solvencia y Liquidez
\end{tabular} & $\begin{array}{l}\mathrm{H}_{1 \mathrm{a}} \\
\mathrm{H}_{2 \mathrm{a}}\end{array}$ & $\begin{array}{l}\text { La influencia de la rentabilidad en la solvencia es similar para las soc. cooperativas y para las no cooperativas. } \\
\text { La influencia de la solvencia en la liquidez es similar para las sociedades cooperativas y para las no cooperativas. }\end{array}$ \\
\hline $\begin{array}{l}\text { Características } \\
\text { de la Empresa }\end{array}$ & $\begin{array}{l}\mathrm{H}_{3 a} \mathrm{yH}_{4 a} \\
\mathrm{H}_{5 a} \mathrm{y}_{6 \mathrm{a}} \\
\mathrm{H}_{7 a} \mathrm{y} \mathrm{H}_{8 \mathrm{a}} \\
\mathrm{H}_{9 \mathrm{a}} \mathrm{y} \mathrm{H}_{10 \mathrm{a}}\end{array}$ & $\begin{array}{l}\text { La influencia de la localización en la solvencia, y la influencia de la localización en la liquidez, es similar para } \\
\text { las sociedades cooperativas y para las no cooperativas. } \\
\text { La influencia del tamaño en la solvencia, y la influencia del tamaño en la liquidez, es similar para las } \\
\text { sociedades cooperativas y para las no cooperativas. } \\
\text { La influencia del sector en la solvencia, y la influencia del sector en la liquidez, es similar para las } \\
\text { sociedades cooperativas y para las no cooperativas. } \\
\text { La influencia de la antigüedad en la solvencia, y la influencia de la antigüedad en la liquidez, es similar para } \\
\text { las sociedades cooperativas y para las no cooperativas. }\end{array}$ \\
\hline $\begin{array}{l}\text { Otros ratios } \\
\text { sobre Liquidez }\end{array}$ & $\begin{array}{l}\mathrm{H}_{11 \mathrm{a}} \\
\mathrm{H}_{12 \mathrm{a}} \\
\mathrm{H}_{13 \mathrm{a}} \\
\mathrm{H}_{14 \mathrm{a}}\end{array}$ & $\begin{array}{l}\text { La influencia de la rotación en la liquidez es similar para las sociedades cooperativas y para las no cooperativas. } \\
\text { La influencia de la estructura económica en la liquidez es similar para las sociedades cooperativas y para las } \\
\text { no cooperativas. } \\
\text { La influencia de la estructura financiera en la liquidez es similar para las soc. cooperativas y para las no cooperativas } \\
\text { La influencia de la actividad en la liquidez es similar para las sociedades cooperativas y para las no cooperativas. }\end{array}$ \\
\hline
\end{tabular}

FUENTE: Elaboración propia. 


\section{3.- Propuesta del modelo teórico}

Como consecuencia de las hipótesis planteadas en el apartado anterior, se obtiene el modelo que se muestra en la Figura 1, que pretende explicar el riesgo de insolvencia empresarial, medido por los efectos sobre la liquidez o solvencia a corto plazo. Dentro de las variables del modelo se distinguen por un lado, los ratios económico-financieros y, por otro, variables de naturaleza no financiera, dado que el fracaso de una empresa es el resultado de un proceso económico complejo con múltiples dimensiones. Los ratios favorecen la adimensionalidad necesaria para evitar los efectos de las unidades de medida, y permitir estudiar la evolución del conjunto de empresas que constituyen la muestra.

\section{Figura 1. Modelo de insolvencia empresarial}

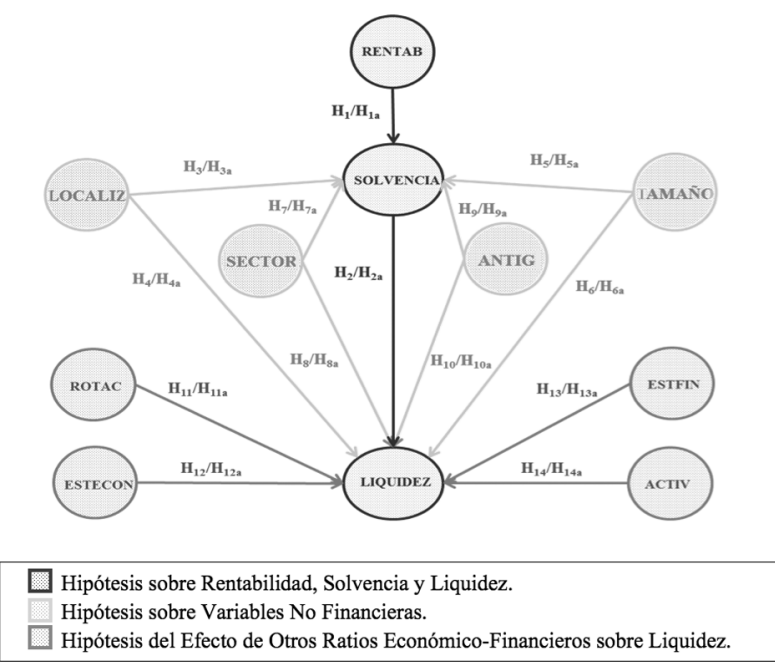

FUENTE: Elaboración propia.

En la elaboración del modelo propuesto se diferencian dos aspectos: por un lado, el universo y la muestra y, por otro, la técnica de análisis.

En lo relativo al universo y la muestra utilizada, la delimitación del concepto de insolvencia empresarial es fundamental para la selección de las empresas que formarán la muestra. En nuestro estudio se ha equiparado a la calificación jurídica de situación concursal, según la Ley Concursal 22/2003², 
de modo que una empresa se considera fracasada si ha presentado un expediente concursal (Deakin, 1972; Lizarraga, 1997). El hecho de optar por el criterio jurídico de situación concursal para la distinción entre una empresa fracasada y una sana dota de una mayor objetividad al estudio, y facilita la fijación de la fecha del fracaso, aunque reduce considerablemente el tamaño de la muestra.

Para la realización del análisis propuesto, se han tomado como referencia las empresas concursadas en España durante el período comprendido entre los años 2005 y 2014. La selección de la muestra parte de los concursos publicados en el Boletín Oficial del Estado durante el periodo objeto de estudio, obtenidos a través de diversas fuentes tales como la WebConcursa/ ${ }^{3}$ y el Registro Público Concursal $^{4}$, que proporcionan datos como la denominación social, el Número de Identificación Fiscal $(\mathrm{NIF})^{5}$, así como la fecha y el registro mercantil en el que se ha presentado el concurso. Los datos contables que se precisaban en el estudio para el cálculo de ratios se han obtenido del Sistema de Análisis de Balances Ibéricos (SABI) 6 .

Para la construcción del modelo se toman los datos económico-financieros (Diéguez et al., 2006, Labatut et al., 2009). Se han utilizado los datos del año previo al concurso, descartando aquellas empresas de reciente creación (hasta dos años), y las que no tenían datos contables completos de hasta al menos dos ejercicios económicos anteriores al fracaso (Pozuelo et al., 2012). Después de este proceso de selección y filtrado, la muestra quedaba reducida a 133 sociedades cooperativas que habían presentado expedientes concursales en el periodo considerado. Para completar la muestra con empresas concursadas de similares características, pero no cooperativas, se recurrió a la técnica de emparejamiento consistente en asociar aleatoriamente 7 a cada una de las sociedades cooperativas quebradas otra de las mismas características, teniendo en cuenta que hubiera entrado en concurso el mismo año, garantizando una correspondencia temporal de sus estados contables, que su tamaño fuera similar, medido en función del volumen de activo empresarial (Pozuelo et al., 2012), y que operase en el mismo sector económico, establecido por la Clasificación Nacional de Actividades Económicas (CNAE), a nivel de dos dígitos. Consecuentemente, la muestra ha quedado formada por 266 empresas, siendo la mitad de ellas cooperativas, y la otra mitad no cooperativas (sociedades anónimas y sociedades limitadas).

3.- Webconcursal, plataforma on-line con información sobre el proceso concursal (https://www.webconcursal.com).

4.- Registro Público Concursal. Ministerio de Justica (https://www.publicidadconcursal.es).

5.- El CIF pasó a denominarse NIF el 1 de enero de 2008, con la entrada en vigor en España del Real Decreto 1065/2007, de 27 de julio, por el que se aprobó el Reglamento General de las actuaciones y los procedimientos de gestión e inspección tributaria y de desarrollo de las normas comunes de los procedimientos de aplicación de los tributos.

6.- $S A B I$, Sistema de Análisis de Balances Ibéricos, Base de Datos, Informa. SABI es una herramienta elaborada por INFORMA D\&B en colaboración con Bureau Van Dijk, que permite manejar la información general y las cuentas anuales de más de 1,25 millones de empresas españolas y más de 500.000 portuguesas. SABI se actualiza periódicamente y la información se obtiene de distintas fuentes oficiales, Registros Mercantiles, BORME, prensa, etc.

7.- Una muestra se considera aleatoria cuando cada elemento presenta la misma probabilidad de ser elegido (lo que asegura su representatividad), y las observaciones se realizan con reemplazamientos (Peña, 2001). La representatividad de la muestra se garantiza si los elementos son elegidos de la forma más objetiva posible para no influir en su selección (Ruiz-Maya y Martín-Pliego, 2005). 
A continuación la siguiente tabla muestra un análisis descriptivo de las empresas que forman parte de la muestra en función de su localización, tamaño, sector y antigüedad:

\section{Tabla 3. Clasificación de las empresas de la muestra}

\begin{tabular}{|c|c|c|c|c|c|}
\hline $\begin{array}{l}\text { LOCALIZACION } \\
\text { (según NUTS) }\end{array}$ & $\begin{array}{l}\text { Sur } \\
48\end{array}$ & \multicolumn{2}{|c|}{$\begin{array}{c}\text { Levante } \\
110\end{array}$} & $\begin{array}{c}\text { Centro } \\
71\end{array}$ & $\begin{array}{c}\text { Norte } \\
37\end{array}$ \\
\hline $\begin{array}{l}\text { TAMAÑO } \\
\text { (volumen Activo) }\end{array}$ & $\begin{array}{c}\text { Microempresa } \\
128\end{array}$ & \multicolumn{2}{|c|}{$\begin{array}{c}\text { Pequeña } \\
108\end{array}$} & $\begin{array}{c}\text { Mediana } \\
24\end{array}$ & $\begin{array}{c}\text { Grande } \\
6\end{array}$ \\
\hline $\begin{array}{l}\text { SECTOR } \\
\text { (según CNAE) }\end{array}$ & $\begin{array}{l}\text { Primario y Extractivas } \\
\qquad 26\end{array}$ & \multicolumn{2}{|c|}{$\begin{array}{c}\text { Industria } \\
104\end{array}$} & $\begin{array}{c}\text { Servicios } \\
96\end{array}$ & $\begin{array}{c}\text { Construción } \\
40\end{array}$ \\
\hline $\begin{array}{l}\text { ANTIGÜEDAD } \\
\text { (años) }\end{array}$ & $\begin{array}{c}\text { Hasta } 5 \\
20\end{array}$ & $\begin{array}{c}\text { Entre } 6 \text { y } 15 \\
82\end{array}$ & $\begin{array}{c}\text { Entre } 16 \text { y } 25 \\
\quad 86\end{array}$ & $\begin{array}{c}\text { Entre } 26 \text { y } 50 \\
\quad 69\end{array}$ & $\begin{array}{c}\text { Más de } 50 \\
9\end{array}$ \\
\hline
\end{tabular}

FUENTE: Elaboración propia.

Dado que en Ciencias Sociales los niveles mínimos de potencia son del 80 por ciento (Cohen, 1988), siendo necesarios cien datos para alcanzar un nivel del 84 por ciento, la muestra utilizada presenta una potencia de estudio elevada, tal y como muestra el Gráfico 1, generado con el Programa G*Power (Faul et al., 2007). Aunque el número de valores perdidos podría alcanzar el 15 por ciento de la muestra (Hair et al., 2014), no se ha registrado la pérdida de ningún valor.

\section{Gráfico 1. Potencia de la muestra}

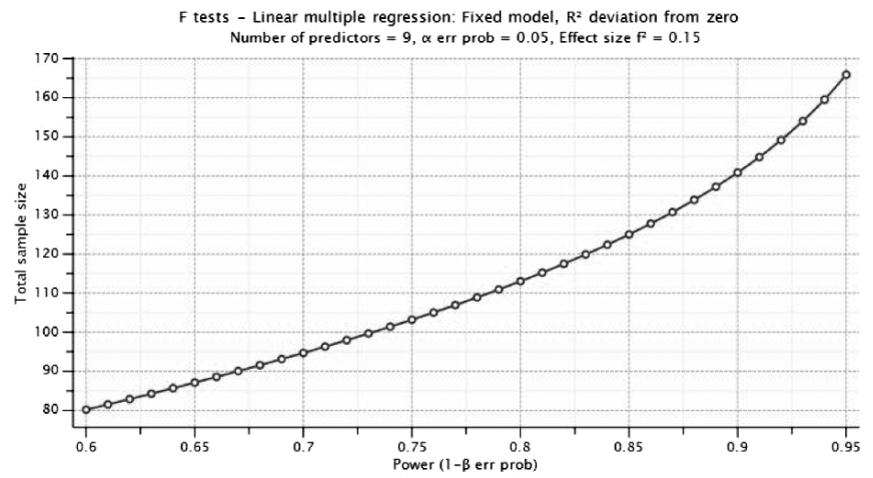

FUENTE: Elaboración propia, a partir del Programa G*Power 3. 
En el análisis de la información económico-financiera extraída de los estados contables de las sociedades cooperativas analizadas hay que tener en cuenta las particularidades de este tipo de sociedades $^{8}$ (Casanellas, 2009, García-Gutiérrez, 2006, Mateos, 2008).

En relación a la técnica de análisis, se ha elaborado un Modelo de Ecuaciones Estructurales basado en Mínimos Cuadrados Parciales (Bagozzi, 1984, Barclay et al., 1995, Bollen, 2014, Cenfetelli y Bassellier, 2009, Cepeda y Roldán, 2004, Chin et al., 2003, Chin y Gopal, 1995, Chin y Newsted, 1999, Gefen et al., 2000, Gerbing y Anderson, 1988, Hair et al., 2006, Hair et al., 2014), denominados Partial Least Square y conocidos como PLS. Su objetivo es la predicción de variables dependientes, maximizando su varianza explicada, por lo que se corresponde con el análisis factorial exploratorio (Chin et al., 2003), y de extracción de componentes principales. Esta técnica se ha popularizado mucho entre los investigadores de ciencias sociales y de administración de empresas debido a las muchas ventajas que presenta frente las técnicas basadas en la covarianza: exigencias de la distribución de las variables de la muestra (no presupone ningún tipo de distribución de los datos, no exigiendo normalidad), el tipo de variables (variables que sean medibles directamente, y variables que no sean observables, ya sean reflectivas o formativas, aumentando la precisión de la estimación), y el tamaño de la propia muestra (de 30 a 100 casos). Dado que el objetivo de PLS es la predicción de las variables dependientes, que no presupone ningún tipo de distribución de los datos y que permite el empleo de variables formativas, resulta imposible mostrar medidas de bondad del ajuste global del modelo (Cepeda y Roldán, 2004). No obstante, es posible el empleo de técnicas no paramétricas de remuestreo para examinar la estabilidad de las estimaciones ofrecidas por PLS. En esta investigación se ha utilizado la técnica de bootstrapping.

La herramienta informática utilizada en la realización de este trabajo es el Programa SmartPLS (Ringle et al., 2015). La ficha técnica de la investigación se recoge en la siguiente Tabla.

\section{Tabla 4. Ficha técnica de la investigación}

\begin{tabular}{|l|l|}
\hline UNIVERSO (POBLACIÓN) & Sociedades Concursadas \\
TAMAÑO MUESTRAL & 266 empresas (133 cooperativas y 133 no cooperativas) \\
OBTENCIÓN DE LA INFORMACIÓN & Páginas Web: Webconcursaly Registro Público Concursal. Base de Datos: SABI \\
HORIZONTE TEMPORAL & $2005-2014$ \\
TÉCNICA DE ANÁLISIS & Modelo de Ecuaciones Estructurales basado en Mínimos Cuadrados Parciales (PLS) \\
SOFTWARE INFORMÁTICO & PLS. Ringle et al., (2015). "SmartPLS 3." Boenningstedt: SmartPLS GmbH, \\
& http://www.smartpls.com \\
\hline
\end{tabular}

FUENTE: Elaboración propia.

8.- La información obtenida de la base de datos $S A B I$ sobre las sociedades cooperativas, ha sido analizada previamente teniendo en cuenta las particularidades de estas sociedades en cuanto a su estructura financiera, así como al reparto de resultados, de manera que la información utilizada en el modelo se permite el análisis conjunto de estas sociedades y de otras formas jurídicas (Martín et al., 2007). 


\section{4.- Selección de las variables explicativas y formulación del modelo}

El modelo teórico planteado incluye como indicadores diversos ratios económico-financieros empleados en estudios anteriores. Para adecuar y confirmar que el modelo propuesto se adapta a la realidad, se ha procedido a realizar previamente un análisis factorial que permite agrupar dichos indicadores en función de la variabilidad que cada uno de ellos comparte con el resto de indicadores afectos al mismo constructo, maximizando la varianza común entre las variables (comunalidad). Además, la utilización de múltiples ratios económico-financieros supone trabajar con una serie de variables que pueden contener información repetida y redundante, lo cual podría causar multicolinealidad, principal problema en la elaboración de los modelos de predicción empresarial. Este hecho se ha evitado al reducir el número de variables económico-financieras, pasando de los 61 indicadores propuestos al inicio del modelo hasta los 22 finales, incluidas otras variables no financieras (ver Tabla 5).

\section{Tabla 5. Relación de indicadores utilizados en el modelo final}

\begin{tabular}{|l|l|l|c|}
\hline CLAVE & DENOMINACIÓN & EXPRESIÓN MATEMÁTICA & FORMULACION \\
\hline ESTEC1 & Peso del Inmovilizado & Activo No Corriente / Activo Total & ANC/A \\
ESTEC3 & Peso del Inmovilizado Material & Inmovilizado Material/ Activo Total & IM/A \\
ESTFI6 & Importancia del Pasivo sobre el Patrimonio Neto & Pasivo / Patrimonio Neto & PNC+PC/PN \\
ESTFI7 & Importancia del Pasivo No Corriente sobre el Patrim Neto & Pasivo No Corriente / Patrimonio Neto & PNC/PN \\
ESTF18 & Importancia del Pasivo Corriente sobre el Patrimonio Neto & Pasivo Corriente / Patrimonio Neto & PC/PN \\
\hline SOL1 & Coeficiente de Garantía & Activo / Pasivo & A/PNC+PC \\
SOL2 & Capacidad de Devolución & Beneficio Antes de Impuestos /Pasivo & BAI/PNC+PC \\
\hline LIQ1 & Ratio de Liquidez & Activo Corriente / Pasivo Corriente & AC/PC \\
LIQ2 & Ratio de Tesorería Ordinaria o Prueba Ácida & (Activo Corriente - Existencias) / Pasivo Corriente & (AC-R)/PC \\
LIQ4 & Peso del Fondo de Maniobra sobre el Pasivo Corriente & Fondo de Maniobra / Pasivo Corriente & FM/PC \\
\hline RENT1 & Rentabilidad Económica (ROA) & Resultado de Explotación/Activo Total & BAll/A \\
RENT3 & Variante Rentabilidad Económica & Resultado del Ejercicio / Activo & BDI/A \\
RENT4 & Rentabilidad Financiera de la Financiación Ajenan & Resultado del Ejercicio / Pasivo & BDI/(PNC+PC) \\
RENT5 & Variante Rentabilidad Económica & Resultado Actividades Ordin después de Impuestos / Activo & BAODI/A \\
RENT6 & Variante Rentabilidad Financiera de la Financiac. Ajena & Resultado Activ. Ordinarias después de Impuestos / Pasivo & BAODI/(PNC+PC) \\
RENT12 & Autofinanc. Generada por ventas & Cash-Flow / Activo & CF/A \\
\hline ACT2 & Tasa de Participac. Gastos de Personal sobre Valor Añadido & Gastos de Personal / Valor Añadido & GP/NA \\
ACT3 & Tasa Participac. Gastos Financieros sobre el Valor Añadido & Gastos Financieros / Valor Añadido & GF/VA \\
ACT5 & Tasa Participac del Gasto de Amortizac. sobre Valor Añadido & Dotación de Amortizaciones / Valor Añadido & AM/VA \\
\hline
\end{tabular}




\begin{tabular}{|l|l|l|c|}
\hline CLAVE & DENOMINACIÓN & EXPRESIÓN MATEMÁTICA & FORMULACION \\
\hline ROT1 & Rotación del Activo & Importe Neto de la Cifra de Ventas / Activo & CNN/A \\
ROT2 & Rotación del Activo Corriente & Importe Neto de la Cifra de Ventas / Activo Corriente & CNN/AC \\
ROT3 & Rotación del Activo No Corriente & Importe Neto de la Cifra de Ventas / Activo No Corriente & CNN/ANC \\
\hline ZONCEN & Empresas sede zona centro & Aragón, Castilla-La Mancha, Castilla y León, La Rioja, Madrid y Navarra & DUMMY \\
ZONLEV & Empresas sede zona levante & Cataluña, Comunidad Valenciana, Islas Baleares y Murcia. & DUMMY \\
ZONSUR & Empresas sede zona sur & Andalucía, Extremad. e Islas Canarias & DUMMY \\
\hline SECTORC & Emp. Sector Construcción & CNAl 41-43 & DUMMY \\
SECTORI & Emp. Sector Industrial & CNAl 10-33 & DUMMY \\
SECTORS & Emp. Sector Servicios & CNAl 45-99 & DUMMY \\
\hline LN_TAMACT & Volumen activo & & LOG ACTIVO \\
LN_TAMEMP & Número empleados & & LOG NEMP \\
LN_TAMING & Ingresos & & LOG ING \\
LN_ANT & Años de vida de la empresa & & LOG ANT \\
\hline
\end{tabular}

FUENTE: Elaboración propia.

La Figura 2 recoge el modelo final con los indicadores incluidos en el mismo.

\section{Figura 2. Modelo que explica la insolvencia empresarial}

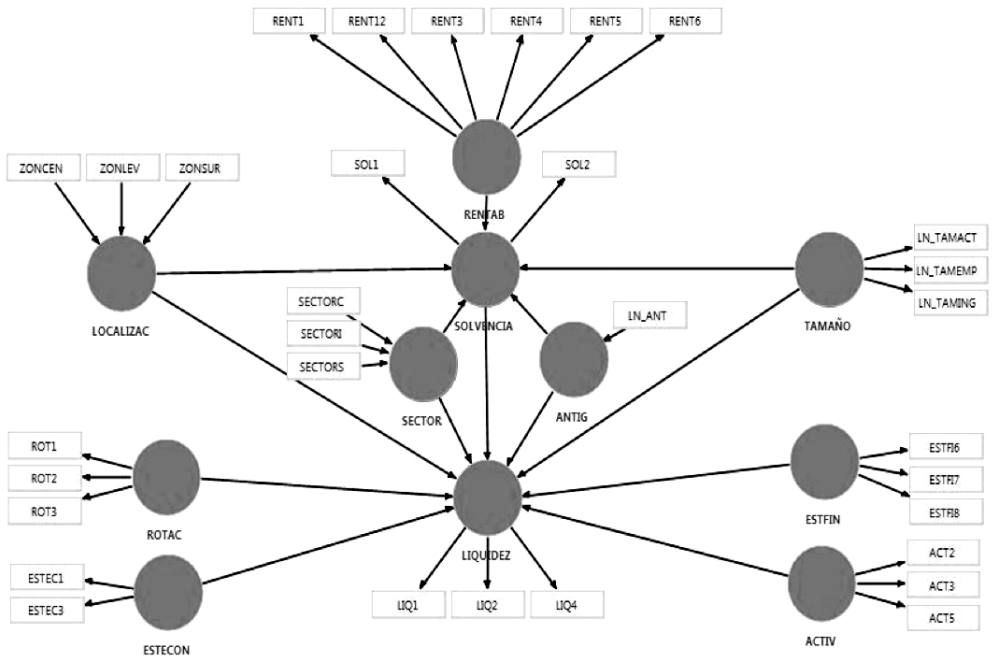

FUENTE: Elaboración propia, utilizando SmartPLS 3. 


\section{5.- Evaluación del modelo}

Los modelos PLS se analizan en dos etapas:

- Valoración del modelo de medida, o modelo externo: relación de cada variable latente con el bloque de variables observables o indicadores afines (Outer Measurement Relations).

- Valoración del modelo estructural, o modelo interno: definido por las relaciones entre las variables latentes dependientes e independientes, representando la teoría y las hipótesis planteadas que hay detrás del modelo (Inner Structural Relations).

El modelo ha sido evaluado atendiendo a los criterios de fiabilidad (ausencia de error, mostrando la precisión del instrumento de medida), y validez (grado con el que un indicador mide aquello que debe medir), a dos niveles (constructo e indicadores asociados), realizándose de diferente manera según las relaciones fueran reflectivas (Tamaño, Rentabilidad, Actividad, Rotación, Estructuras Económica y Financiera, o formativas (Sector, Localización y Antigüedad).

Asimismo, se estudia si existe un efecto moderador de la forma jurídica en la liquidez y en la solvencia de las sociedades analizadas. Para ello, se ha realizado un Análisis Multigrupo, aplicando el Enfoque No Paramétrico o PLS MGA (Henseler et al., 2009). Se ha codificado la forma jurídica como una variable dummy dicotómica, presentando valor 1 para sociedades cooperativas y valor 0 para sociedades no cooperativas.

En lo relativo al estudio de la significatividad de las hipótesis planteadas en el modelo, tanto para la totalidad de empresas concursadas, independientemente de su forma jurídica, como para los dos grupos diferenciados (sociedades cooperativas y sociedades no cooperativas), se ha implementado un procedimiento de bootstrapping, separándose los resultados relacionados con las hipótesis de las relaciones entre rentabilidad, solvencia y liquidez, las hipótesis vinculadas a las variables sobre las características de la empresa y, por último, las hipótesis relativas al efecto sobre la liquidez del resto de variables económico-financieras.

\section{A) MODELO EXTERNO}

Dentro de este modelo se analiza:

- La fiabilidad y validez de los indicadores de los constructos reflectivos: se contrasta comprobando que las cargas factoriales de los indicadores (outer loadings) son todas superiores al umbral mínimo de 0,7 . 
- La fiabilidad y validez de los constructos reflectivos: se evalúa la fiabilidad de los constructos reflexivos mediante el Alpha de Cronbach y la Fiabilidad Compuesta, mientras que la validez se analiza con la Average Variance Extracted (AVE). En la Tabla 6 se comprueba que en todos los casos superan los criterios mínimos. Además, también cumplen el Criterio de FornellLarcker y la Ratio Heterotrait-Monotrait (HT/MT). Asimismo, todos los indicadores presentan un mayor efecto en su variable latente asociada en la Matriz de Cargas Cruzadas.

Sobre la base de los criterios expuestos con anterioridad, se concluye que los constructos reflectivos, y sus indicadores asociados, son un instrumento de medida fiable y válido.

\section{Tabla 6. Fiabilidad y validez de los constructos reflexivos}

\begin{tabular}{|lccc|}
\hline VARIABLE & ALPHA CRONBACH & FIABILIDAD COMPUESTA & AVE \\
\hline ACTIV & 0,862 & 0,906 & 0,764 \\
ESTECON & 0,836 & 0,924 & 0,859 \\
ESTFIN & 0,981 & 0,986 & 0,960 \\
LIQUIDEZ & 0,884 & 0,928 & 0,812 \\
RENTAB & 0,930 & 0,932 & 0,697 \\
ROTAC & 0,880 & 0,895 & 0,741 \\
SOLVENCIA & 0,613 & 0,834 & 0,717 \\
TAMAÑO & 0,867 & 0,918 & 0,790 \\
\hline
\end{tabular}

Estimaciones obtenidas con SmartPLS 3. Modelo de Ecuaciones Estructurales basados en Mínimos Cuadrados Parciales.

- La significatividad de los indicadores de los constructos reflectivos: para estimar la significatividad de estos parámetros se ha utilizado el denominado bootstrapping, generando 5.000 muestras del mismo tamaño que la original (266 observaciones). Tras los $p$-valores obtenidos, se pone de manifiesto que todos los indicadores reflectivos son significativos al $1 \%$.

- La multicolinealidad de los indicadores de los constructos formativos: el diagnóstico de la multicolinealidad se lleva a cabo por el Factor de Inflación de la Varianza (VIF), el cual debe ser inferior a 5 (Hair et al., 2011), cumpliéndose esta premisa en el modelo planteado, tal y como recoge la siguiente Tabla. 


\section{Tabla 7. Factor de inflación de la varianza de los indicadores formativos (VIF)}

\begin{tabular}{|lc|}
\hline INDICADOR & VIF \\
\hline LN_ANT & 1,000 \\
SECTORC & 2,157 \\
SECTORI & 3,045 \\
SECTORS & 2,999 \\
ZONCEN & 2,140 \\
ZONLEV & 2,330 \\
ZONSUR & 1,883 \\
\hline
\end{tabular}

Estimaciones obtenidas con SmartPLS 3. Modelo de Ecuaciones Estructurales basados en Mínimos Cuadrados Parciales.

- La significatividad de los indicadores de los constructos formativos: para determinar si los pesos de los indicadores formativos son estadísticamente significativos se aplica el procedimiento bootstrapping. El resultado es que el Sector de Construcción y Zona Sur son significativos con un intervalo de confianza del $95 \%$. No obstante, se decide no eliminar los restantes indicadores dada su importancia de contenido para la investigación.

\section{B) MODELO INTERNO}

Dentro de este modelo se analiza:

- Multicolinealidad en el Modelo Estructural: Se evalúa mediante el Factor de Inflación de la Varianza (VIF), aplicándose a los dos submodelos de Liquidez y Solvencia. El resultado obtenido muestra el cumplimiento del criterio para ambos submodelos analizados.

- Precisión de las Predicciones (indicador $R^{2}$ ): El coeficiente de determinación $\left(R^{2}\right)$ indica la cantidad de varianza de las variables latentes endógenas, en nuestro caso Solvencia y Liquidez, explicada por el modelo planteado. Se obtiene una explicación de 36,2\% de la liquidez y del $54,7 \%$ de la solvencia. El indicador $\left(R^{2}\right)$ nunca debe ser inferior a 0,1 (Falk y Miller, 1992), valor mínimo que se supera a la vista de los resultados recogidos.

\section{Tabla 8. Indicador $\left(R^{2}\right)$ y $\left(R^{2}\right)$ ajustado}

\begin{tabular}{|lcc|}
\hline VARIABLES & $\mathbf{R}^{\mathbf{2}}$ & $\mathbf{R}^{\mathbf{2}}$ AJUSTADO \\
\hline LIQUIDEZ & 0,383 & 0,362 \\
SOLVENCIA & 0,555 & 0,547 \\
\hline
\end{tabular}

Estimaciones obtenidas con SmartPLS 3. Modelo de Ecuaciones Estructurales basados en Mínimos Cuadrados Parciales. 
- Tamaño de los Efectos f2: mide los efectos de un constructo exógeno en un constructo endógeno mediante el cambio que se produce en la $R^{2}$ del constructo endógeno al excluir el exógeno. El resultado es un efecto reducido en las relaciones entre el Sector de Actividad al que pertenece la empresa, y la Estructura Económica, con respecto a la variable Liquidez. Sin embargo, tiene especial relevancia el efecto de la variable Solvencia sobre Liquidez y, sobre todo, el de la variable Rentabilidad sobre Solvencia.

\section{Tabla 9. Tamaño de los efectos (Indicador $\mathbf{f}^{2}$ )}

\begin{tabular}{|ll|}
\hline ACTIV-LIQUIDEZ & 0,002 \\
ANTIG-LIQUIDEZ & 0,002 \\
ANTIG-SOLVENCIA & 0,013 \\
ESTECON-LIQUIDEZ & 0,057 \\
ESTFIN-LIQUIDEZ & 0,001 \\
LOCALIZAC-LIQUIDEZ & 0,000 \\
LOCALIZAC-SOLVENCIA & 0,006 \\
RENTAB-SOLVENCIA & 1,136 \\
ROTAC-LIQUIDEZ & 0,000 \\
SECTOR-LIQUIDEZ & 0,016 \\
SECTOR-SOLVENCIA & 0,001 \\
SOLVENCIA-LIQUIDEZ & $\mathbf{0 , 4 5 2}$ \\
TAMAÑO-LIQUIDEZ & 0,005 \\
TAMAÑO-SOLVENCIA & 0,003 \\
\hline
\end{tabular}

Estimaciones obtenidas con SmartPLS 3. Modelo de Ecuaciones Estructurales basados en Mínimos Cuadrados Parciales.

- Relevancia Predictiva del Modelo mediante el Estadístico Q2: para medir la relevancia predictiva de las variables latentes endógenas se utiliza el estadístico $\left(Q^{2}\right)$ (Geisser, 1975, Stone, 1974). Se calcula utilizando el procedimiento blindfolding, que consiste en omitir parte de los datos cuando se estima una variable latente dependiente a partir de otras independientes, para luego estimar esos datos utilizando los parámetros predichos (Chin, 1998, Henseler et al., 2015). Utilizando una distancia de omisión de 6 , se obtiene una relevancia predictiva elevada de la Solvencia $(0,372)$, y media de la Liquidez $(0,241)$.

C) SIGNIFICATIVIDAD ESTADÍSTICA: RELEVANCIA DE LAS HIPÓTESIS PLANTEADAS Y EL POSIBLE EFECTO MODERADOR DE LA FORMA JURÍDICA

Para su estudio se utiliza de nuevo el procedimiento de bootstrapping obteniendo los resultados que aparecen en la Tabla 10. 
Tabla 10. Significatividad estadística de las hipótesis

\begin{tabular}{|c|c|c|c|c|}
\hline HIPÓTESIS & $\begin{array}{l}\text { MUESTRA } \\
\text { ORIGINAL }\end{array}$ & $\begin{array}{l}\text { VALOR ORIGINAL } \\
\text { (COOP) }\end{array}$ & $\begin{array}{l}\text { VALOR ORIGINAL } \\
\text { (NO COOP) }\end{array}$ & $\begin{array}{c}\text { DIFERENCIAS } \\
\text { (COOP - NO COOP) }\end{array}$ \\
\hline $\mathrm{H}_{1} / \mathrm{H}_{1 \mathrm{a}}:$ RENTAB $>$ SOLVENCIA & $0,737^{\star \star \star}$ & $0,691^{* \star *}$ & $0,772^{\star \star *}$ & 0,081 \\
\hline $\mathrm{H}_{2} / \mathrm{H}_{2 \mathrm{a}}:$ SOLVENCIA $>$ LIQUIDEZ & $0,552^{\star \star *}$ & $0,668^{\star \star *}$ & $0,392^{\star *}$ & 0,276 \\
\hline $\mathrm{H}_{3} / \mathrm{H}_{3 \mathrm{a}}:$ LOCALIZAC $->$ SOLVENCIA & 0,053 & $0,171^{\star *}$ & 0,042 & $0,129^{*}$ \\
\hline $\mathrm{H}_{4} / \mathrm{H}_{4 \mathrm{a}}:$ LOCALIZAC -> LIQUIDEZ & $-0,003$ & $-0,028$ & $-0,031$ & 0,003 \\
\hline $\mathrm{H}_{5} / \mathrm{H}_{5 \mathrm{a}}:$ TAMAÑO -> SOLVENCIA & $-0,039$ & $-0,144^{*}$ & 0,058 & 0,202 \\
\hline $\mathrm{H}_{6} / \mathrm{H}_{6 \mathrm{a}}:$ TAMAÑO -> LIQUIDEZ & $-0,060$ & $-0,117^{\star}$ & 0,004 & 0,120 \\
\hline $\mathrm{H}_{7} / \mathrm{H}_{7 \mathrm{a}}: \mathrm{SECTOR}$-> SOLVENCIA & $-0,023$ & 0,047 & 0,048 & 0,001 \\
\hline $\mathrm{H}_{8} / \mathrm{H}_{8 \mathrm{a}}:$ SECTOR -> LIQUIDEZ & $0,105^{\star}$ & 0,096 & 0,141 & 0,045 \\
\hline $\mathrm{Hg}_{g} / \mathrm{H}_{9 \mathrm{a}}:$ ANTIG $->$ SOLVENCIA & $0,080^{*}$ & $0,105^{\star *}$ & $-0,036$ & $0,140^{* *}$ \\
\hline $\mathrm{H}_{10} / \mathrm{H}_{10 \mathrm{a}}:$ ANTIG -> LIQUIDEZ & 0,038 & 0,040 & 0,055 & 0,015 \\
\hline $\mathrm{H}_{11} / \mathrm{H}_{11 \mathrm{a}}:$ ROTAC -> LIQUIDEZ & $-0,002$ & $-0,012$ & $-0,030$ & 0,018 \\
\hline $\mathrm{H}_{12} / \mathrm{H}_{12 \mathrm{a}}$ : ESTECON -> LIQUIDEZ & $-0,194^{\star \star *}$ & $-0,190^{\star \star *}$ & $-0,278^{\star \star *}$ & 0,088 \\
\hline $\mathrm{H}_{13} / \mathrm{H}_{13 \mathrm{a}}:$ ESTFIN -> LIQUIDEZ & $-0,024$ & 0,000 & $0,144^{\star *}$ & 0,144 \\
\hline $\mathrm{H}_{14} / \mathrm{H}_{14 \mathrm{a}}:$ ACTIV -> LIQUIDEZ & $-0,037$ & $-0,004$ & $-0,066$ & 0,062 \\
\hline
\end{tabular}

Estimaciones obtenidas con SmartPLS 3. Modelo de Ecuaciones Estructurales basados en Mínimos Cuadrados Parciales. Los asteriscos recogen el nivel de significación de rechazo de la hipótesis nula: $1 \%{ }^{* *}, 5 \%{ }^{* *}, 10 \%{ }^{*}$.

Del contraste de las hipótesis planteadas en el modelo para la totalidad de empresas concursadas subyace que la Rentabilidad influye positiva y significativamente sobre la Solvencia, al igual que la Solvencia sobre la Liquidez, evidenciándose una gran correlación entre los indicadores de solvencia a largo plazo y a corto plazo. Por otro lado, los indicadores de Estructura Económica seleccionados tendrían un pequeño efecto significativo negativo sobre la Liquidez. Dentro de las variables no financieras solo son significativas, con un menor nivel, por un lado la Antigüedad sobre la Solvencia y por otro el Sector sobre la Liquidez.

Si se realiza el contraste para cada una de las submuestras, los resultados evidencian algunas diferencias de las sociedades cooperativas en las hipótesis relativas a los efectos de las variables no financieras en las características de la empresa sobre la Solvencia y la Liquidez. Estas diferencias son analizadas en las conclusiones:

- La Localización tiene una influencia positiva en la Solvencia de las sociedades, tanto cooperativas, como no cooperativas, siendo solo significativa en las primeras. 
- El Tamaño presenta una pequeña correlación negativa y marginalmente significativa en la Solvencia y la Liquidez de las sociedades cooperativa, frente a un efecto positivo, pero no significativo, en las sociedades de diferente forma jurídica.

- La Antigüedad tiene un pequeño efecto positivo y significativo sobre la Solvencia de las sociedades cooperativas.

- No ha resultado significativa la influencia de la variable de Estructura Financiera en la Liquidez de las sociedades cooperativas. No obstante, sí es observable un efecto positivo y significativo para las sociedades no cooperativas.

Sin embargo, no todas estas diferencias son significativas. Se puede afirmar que la forma jurídica modera únicamente la influencia de los efectos de la Localización y la Antigüedad sobre la Solvencia, con un nivel de significación del 10 por ciento y del 5 por ciento, respectivamente, no encontrándose diferencias en el resto de variables analizadas.

La Figura 3 muestra la carga de los indicadores asociados a variables latentes reflectivas (Solvencia, Liquidez, Rentabilidad, Actividad, Rotación, Estructura Económica, Estructura Financiera y Tamaño), el peso de los indicadores asociados a variables latentes formativas (Sector, Localización y Antigüedad), el valor de los parámetros que relacionan las variables latentes (Hipótesis Planteadas), así como la precisión de las predicciones (Indicador $R^{2}$ ).

\section{Figura 3. Resultados del modelo propuesto}

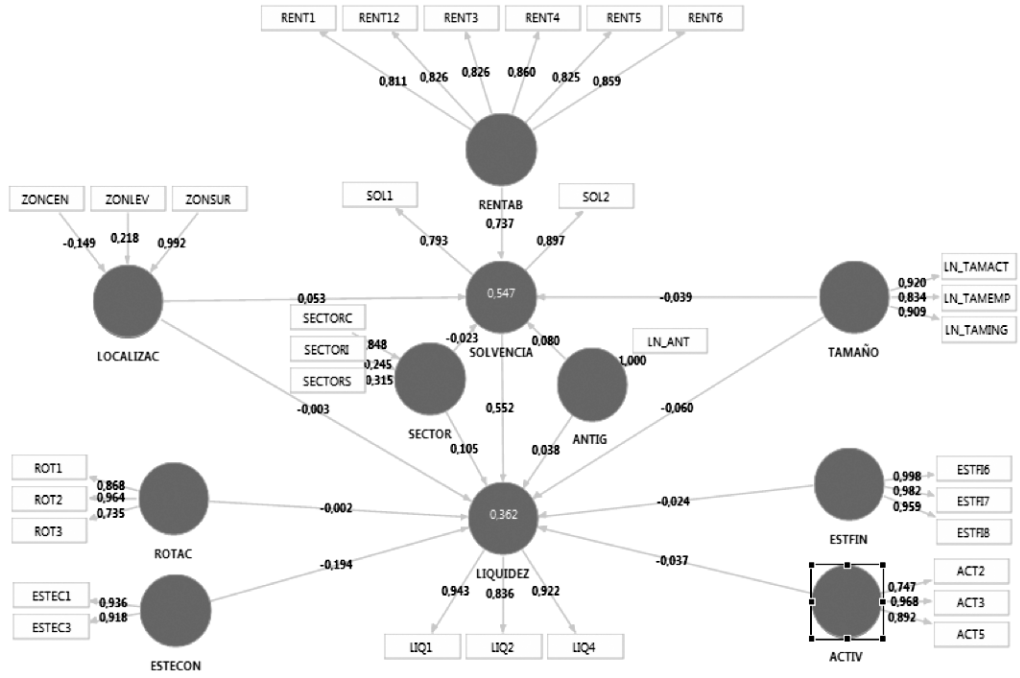

Estimaciones obtenidas con SmartPLS 3. Modelo de Ecuaciones Estructurales. 
Teniendo en cuenta los resultados conseguidos, tanto los constructos endógenos, como sus indicadores asociados, conforman un instrumento de medida fiable y válido, alcanzando y superando los valores mínimos deseables de los indicadores de fiabilidad (cargas y pesos factoriales, Alpha de Cronbach, fiabilidad compuesta), y validez (varianza extraída media, factor de inflación de la varianza), detallados en esta investigación, tanto para la totalidad de la muestra, como para ambas submuestras.

La precisión de las predicciones, medida a través del coeficiente de determinación $\left(R^{2}\right)$ de las variables Solvencia y Liquidez es satisfactoria, a tenor de los resultados alcanzados en dicho coeficiente. Por otro lado, a través del indicador $\left(\mathrm{f}^{2}\right)$ se pone de manifiesto la especial relevancia del efecto que tiene la variable Solvencia sobre Liquidez y, sobre todo, la variable Rentabilidad sobre Solvencia. Asimismo, el estadístico $\left(Q^{2}\right)$ muestra que el modelo tiene capacidad predictiva para ambas variables, al ser el resultado mayor que cero.

\section{6.- Conclusiones}

En el modelo planteado se evalúa tanto la fiabilidad como la validez de las variables e indicadores asociados, y teniendo en cuenta los resultados conseguidos, todos conforman un instrumento de medida fiable y válido, alcanzando y superando los valores mínimos deseables. Asimismo, todos los indicadores reflectivos son estadísticamente significativos al 1\%, mientras que en el caso de los formativos destaca la significatividad del Sector de la Construcción y de la localización en la Zona Sur.

El modelo propuesto de insolvencia empresarial se considera preciso para anticipar estas situaciones, dado que el coeficiente de determinación $\left(R^{2}\right)$ ajustado presenta un valor satisfactorio, superando con creces el valor mínimo deseable en las variables analizadas: la Solvencia es explicada en un $54 \%$, y la Liquidez en un $36 \%$. Por otro lado, la relevancia predictiva es elevada para la variable Solvencia, y media para la variable Liquidez.

En primer lugar se exponen las conclusiones relativas al Modelo Global, teniendo en cuenta la totalidad de las empresas que conforman la muestra. A continuación se detallan las peculiaridades más significativas para las sociedades cooperativas. 


\section{A) Conclusiones sobre las Hipótesis Planteadas en el Modelo Global:}

- La Rentabilidad de las empresas, previa a la entrada en el proceso concursal, afecta de forma positiva y significativa a la Solvencia y ésta, a su vez, a la Liquidez. Consecuentemente, una mayor rentabilidad permite a las empresas mayor solvencia evitando los problemas de liquidez que llevan al concurso de acreedores y, por tanto, al fracaso empresarial.

- La Estructura Económica de la empresa afecta de forma significativa, aunque en sentido contrario, a la Liquidez. Esta relación puede deberse a que cuanto menor sea la inversión de las empresas en activo fijo aumenta la cantidad de recursos de los que dispone la empresa y, con ello, su liquidez.

- Dentro de las variables no financieras solo son significativas, con un menor nivel, por un lado la Antigüedad sobre la Solvencia y, por otro, el Sector sobre la Liquidez. La primera de las relaciones puede explicarse por una mayor capacidad para generar fondos por parte de las empresas más antiguas. En cuanto al sector, se pone de manifiesto que la práctica habitual en determinadas actividades hace que las empresas puedan tener más liquidez normalmente derivada de la política de cobros y pagos aplicada.

\section{B) Conclusiones sobre el Efecto Moderador de la Forma Jurídica Cooperativa:}

Al realizar el contraste de hipótesis para cada una de las submuestras, los resultados evidencian algunas diferencias para las sociedades cooperativas en las hipótesis relativas a los efectos de las variables relativas a las características de las empresas sobre la Solvencia y la Liquidez, no siendo todas ellas significativas:

- La Localización tendría una influencia positiva y significativa en las sociedades cooperativas. Sin embargo, la ubicación influye positiva, pero no significativamente, en las entidades de diferente forma jurídica, a excepción de la Zona de Levante.

- Existe una pequeña correlación negativa, y marginalmente significativa, entre la Dimensión y la Solvencia de las sociedades cooperativas, al igual que con su liquidez, frente a un efecto positivo pero no significativo, en las sociedades de diferente forma jurídica.

- Se produce un pequeño efecto positivo y significativo de la Antigüedad de las sociedades cooperativas sobre la Solvencia de las mismas, consecuencia de la dotación anual obligatoria de recursos que realizan a los fondos de reserva obligatorios.

- Por otro lado, no ha resultado significativa la influencia de la variable de Estructura Financiera en la Liquidez de las sociedades cooperativas, representada principalmente por el indicador de endeudamiento asociado a dicha variable. Sin embargo, si es observable un efecto positivo y significativo para las sociedades no cooperativas. 
- En relación a las diferencias enunciadas entre las sociedades cooperativas y no cooperativas analizadas, únicamente las influencias de la Localización y la Antigüedad sobre la Solvencia resultan significativas.

En definitiva, la Rentabilidad, la Solvencia y la Estructura Económica anticipan posibles situaciones de fracaso empresarial. Las dos primeras tienen un efecto positivo sobre la solvencia a largo y a corto plazo, corroborando las hipótesis planteadas en la bibliografía consultada, mientras que la segunda presentaría un efecto negativo, evidenciándose que un mayor resultado en estos indicadores implica un menor peso del activo corriente, alcanzado una menor liquidez.

Al analizar la aplicación del modelo a las sociedades cooperativas se comprueba que las tres variables mencionadas son válidas y significativas para esta forma jurídica, por lo que el modelo también es aplicable a las mismas. No obstante, aparecen cuatro factores diferenciadores: la Localización, la Antigüedad, el Tamaño y la Estructura Financiera, presentando diferencias significativas solo en los dos primeros:

- La Localización tiene una influencia positiva en la solvencia de las sociedades, tanto cooperativas, como no cooperativas, siendo solo significativa en las primeras. Esta divergencia se deriva del análisis de los pesos de las diferentes zonas en ambas submuestras. Se pone en evidencia que la localización en la Zona de Levante, en la que se ubican un considerable número de empresas muestrales de ambos tipos es la única que presenta una considerable influencia negativa en el caso de las no cooperativas, a diferencia del resto de zonas.

- Se aprecia un pequeño efecto positivo y significativo de la Antigüedad sobre la Solvencia de las sociedades cooperativas. Estas sociedades, a diferencia de otras formas jurídicas, tienen que realizar, anual y obligatoriamente, una dotación de recursos al Fondo de Reserva Obligatorio, no existiendo una limitación o tope máximo de la cuantía de estos fondos. Sin embargo, en las sociedades limitadas o anónimas la obligación de destinar el $10 \%$ del beneficio del ejercicio a la reserva legal, sólo se produce hasta que ésta alcanza el $20 \%$ del capital social.

- No ha resultado significativa la influencia de la variable de Estructura Financiera en la Liquidez de las sociedades cooperativas. Sin embargo, si es observable un efecto positivo y significativo para las sociedades no cooperativas. Habitualmente, estas sociedades cuentan con más facilidades para el acceso al crédito en los mercados financieros, con respecto a las sociedades cooperativas, reduciendo su liquidez. 


\section{7.- Bibliografía}

AGUIAR, I. \& RUÍZ, M.V. (2014): "La resolución del concurso en las empresas canarias", Hacienda Canaria, 41, 85-110.

ALFARO, E., GÁMEZ, M. \& GARCÍA, N. (2008): "FIAMM return persistence analysis and the determinants of the fees charged", Spanish Journal of Finance and Accounting/Revista Española de Financiación \& Contabilidad, 137, 13-32.

ALTMAN, E.I. (1968): "Financial ratios, discriminant analysis and the prediction of corporate bankruptcy", The Journal of Finance, 4, 589-609.

ALTMAN, E.I., HALDEMAN, R.G. \& NARAYANAN, P. (1977): "ZETA TM analysis: A new model to identify bankruptcy risk of corporations", Journal of Banking and Finance, 1, 29-54.

BAGOZZI, R.P. (1984): "Expectancy-value attitude models an analysis of critical measurement issues", International Journal of Research in Marketing, 4, 295-310.

BARCLAY, D., HIGGINS, C. \& THOMPSON, R. (1995): "The partial least squares (PLS) approach to causal modeling: Personal computer adoption and use as an illustration", Technology Studies, 2, 285-309.

BARRON, D., WEST, E. \& HANNAN, M. (1994): "A Time to Grow and a Time to Die: Growth and Mortality of Credit Unions in New York City, 1914-1990", American Journal of Sociology, 100, 381421.

BEAVER, W.H. (1966): "Financial ratios as predictors of failure", Journal of Accounting Research, 71-111.

BOLLEN, K.A. (2014): Structural equations with latent variables, John Wile \& Sons, New York.

BRUDERL, J. \& SCHUSSLER, R. (1990): "Organizational Mortality: The Liabilities of Newness and Adolescence", Administrative Science Quarterly, 3, 530-547.

CALDERÓN, B. \& CALDERÓN, M.J. (2012): "Cómo afrontan la crisis las cooperativas en España: comparativa de trayectorias laborales a partir de la Muestra Continua de Vidas Laborales" CIRIECEspaña, Revista de Economía Pública, Social y Cooperativa, 76, 5-26.

CALVO-FLORES, A., GARCÍA, D. \& MADRID, A. (2006): "Tamaño, Antigüedad y Fracaso Empresarial", Working Paper, Cartagena: Universidad Politécnica de Cartagena.

CARROLL, G.R. (1983): "A stochastic model of organizational mortality: Review and reanalysis", Social Science Research, 4, 303-329. 
CASANELLAS, R. (2009): "El nuevo plan general de contabilidad y el concurso de acreedores", Revista de Derecho Concursal y Paraconcursal: Anales de Doctrina, Praxis, Jurisprudencia y Legislación, 11, 395-401.

CASEY, C. \& BARTCZAK, N. (1985): "Using operating cash flow data to predict financial distress: some extensions", Journal of Accounting Research, 23, 384-401.

CENFETELLI, R. T \& BASSELLIER, G. (2009): "Interpretation of formative measurement in information systems research", Mis Quarterly, 33, 689-707.

CEPEDA, G. \& ROLDÁN, J.L. (2004): "Aplicando la técnica PLS en la administración de empresas", En: Conocimiento y competitividad, ACEDE, 14, Murcia.

CHAVES, R. \& MONZÓN, J.L. (2007): La Economía Social en la Unión Europea, Comité Económico y Social Europeo, Bruselas.

CHEN, R. \& WONG, K. (2004): "The Determinants of Financial Health of Asian Insurance Companies", Ann. Journal of Risk and Insurance, 71, 469.

CHIN, W.W. (1998): "Issues and opinion on structural equation modeling", MIS Quarterly, 22, VII.

CHIN, W.W. \& NEWSTED, P.R. (1999): Structural equation modeling analisis with small samples using partial least squares, Sage Publications, Thousand Oaks.

CHIN, W.W. \& GOPAL, A. (1995): "Adoption intention in GSS: relative importance of beliefs", ACM SigMIS Database, 26, 42-64.

CHIN, W.W., MARCOLIN, B.L. \& NEWSTED, P.R. (2003): "A partial least squares latent variable modeling approach for measuring interaction effects: Results from a Monte Carlo simulation study and an electronic-mail emotion/adoption study", Information Systems Research, 14, 189-217.

CLEMENTE, J., DÍAZ-FONCEA, M., MARCUELLO, C. \& SANSO-NAVARRO, M. (2012): "The wage gap between cooperative and capitalism firms: evidence from Spain", Annals of Public and Cooperative Economics, 83, 337-356.

COHEN, J. (1988): Statistical power analysis for the behavioral sciences, 2nd Ed: Hillsdale, Erlbaum.

CORREA, A., ACOSTA, M. \& GONZÁLEZ, A.L. (2003): "La insolvencia empresarial: un análisis empírico para la pequeña y mediana empresa", Revista de Contabilidad, 12, 47-79.

DEAKIN, E.B. (1972): "A discriminant analysis of predictors of business failure", Journal of Accounting Research, 10, 167-179.

DIÉGUEZ, J., TRUJILLO, F. \& CISNEROS, A.J. (2006): "Modelos de predicción de la insolvencia empresarial: la incorporación de ratios a partir de un marco teórico". En: VI Jornadas sobre Predicción del Insolvencia Empresarial, AECA, Carmona (Sevilla).

DIETRICH, J., ARCELUS, F. \& SRINIVASAN, G. (2005): "Predicting financial failure: some evidence from new brunswick agricultural co ops", Annals of Public and Cooperative Economics, 76, 179-194. 
DOMINGO, J. (2001): "Ratios para el análisis de rentabilidad de las cooperativas agrarias andaluzas", CIRIEC-España, Revista de Economía Pública, Social y Cooperativa, 38, 171-186.

DUNNE, T., ROBERTS, M.J. \& SAMUELSON, L. (1988): "Patterns of firm entry and exit in US manufacturing industries", The RAND Journal of Economics, 19, 495-515.

FAUL, F., ERDFELDER, E., LANG, A.G. \& BUCHNER, A. (2007): "G* Power 3: A flexible statistical power analysis program for the social, behavioral, and biomedical sciences", Behavior Research Methods, 39, 175-191.

FAJARDO, G. (2011): "How viable are Spanish Credit Cooperatives after recent bank capitalization and restructuring regulations?", CIRIEC-España, Revista de Economía Pública, Social y Cooperativa, 73, 151-170

FALK, R.F. \& MILLER, N.B. (1992): A primer for soft modeling, Akron, Ohio: University of Akron Press.

FERRANDO, M. \& BLANCO, F. (1998): "La Previsión del Fracaso Empresarial en la Comunidad Valenciana: Aplicación de los Modelos Discriminante y Logit", Revista Española de Financiación y Contabilidad, 95, 499-540.

FITZPATRICK, P. (1932): "A Comparison of ratios of successful industrial enterprises with those of failed firms", Certified Public Accountant, 12, 598-605, 656-662 \& 727-731.

GABÁS, F. (1990): Técnicas actuales de análisis contable: evaluación de la solvencia empresarial, Instituto de Contabilidad y Auditoría de Cuentas, Ministerio de Economía y Competitividad, Madrid.

GALINDO, A. (2006): "Repercusiones de la definición de tamaño empresarial en los resultados empíricos sobre eficiencia y financiación", Observatorio Iberoamericano del Desarrollo Local y la Economía Social, 1, 308-361.

GARCÍA-GUTIÉRREZ, C. (2006): "La reafirmación de las aportaciones de (propiedad de) los socios de las sociedades cooperativas, propuesta de regulación de las sociedades de responsabilidad limitada cooperativa", REVESCO, Revista de Estudios Cooperativos, 89, 27-83.

GEFEN, D., STRAUB, D. \& BOUDREAU, M.C. (2000): "Structural equation modeling and regression: Guidelines for research practice", Communications of the Association for Information Systems, 4,7 .

GEISSER, S. (1975): "The predictive sample reuse method with applications", Journal of the American Statistical Association, 350, 320-328.

GENTRY, J., NEWBOLD, P. \& WHITFORD, D. (1985): "Classifying bankrupt firms with funds flow components", Journal of Accounting Research, 2, 146-160.

GENTRY, J., NEWBOLD, P. \& WHITFORD, D. (1987): "Funds flow components, financial ratios, and bankruptcy", Journal of Business Finance and Accounting, 14, 595-606. 
GERBING, D.W. \& ANDERSON, J.C. (1988): "An updated paradigm for scale development incorporating unidimensionality and its assessment", Journal of Marketing Research, 25, 186-192.

GÓMEZ-LIMÓN, J.A., CASQUET, E. \& ATANCE, I. (2003): "Análisis económico-financiero de las cooperativas agrarias en Castilla y León", CIRIEC-España, Revista de Economía Pública, Social y Cooperativa, 46, 151-189.

GÓMEZ, M.E., DE LA TORRE, J.M. \& ROMÁN, I. (2008). Análisis de sensibilidad temporal en los modelos de predicción de insolvencia: una aplicación a las PYMES industriales, Spanish Journal of Finance and Accounting/Revista Española de Financiación y Contabilidad, 137, 85-111.

HAIR J.F., HULT, G.T.M., RINGLE, C. \& SARSTEDT, M. (2014): A primer on partial least squares structural equation modeling (PLS-SEM), Sage Publications, Thousand Oaks.

HAIR, J.F., BLACK, W.C., BABIN, B.J., ANDERSON, R.E. \& TATHAM, R.L. (2006): Multivariate data analysis, $\sigma^{a}$ ed: Prentice-Hall International, New Jersey.

HAIR, J.F., RINGLE, C.M. \& SARSTEDT, M. (2011): "PLS-SEM: indeed a silver bullet", Journal of Marketing Theory and Practice, 19, 139-152.

HELLMANN, T. \& STIGLITZ, J. (2000): "Credit and equity rationing in markets with adverse selection", European Economic Review, 44, 281-304.

HENDERSON, A.D. (1999): "Firm strategy and age dependence: A contingent view of the liabilities of newness, adolescence, and obsolescence", Administrative Science Quarterly, 44, 281-314.

HENSELER, J. RINGLE, C.M. \& SARSTEDT, M. (2015): "A new criterion for assessing discriminant validity in variance-based structural equation modeling", Journal of the Academy of Marketing Science, 43, 115-135.

HENSELER, J., RINGLE, C.M. \& SINKOVICS, R.R. (2009): "The use of partial least squares path modeling in international marketing", Advances in International Marketing (AIM), 20, 277-320.

ITURRIOZ, J. (2010): "Los procesos concursales en situación de crisis: características de su aplicación a las sociedades cooperativas", REVESCO, Revista de Estudios Cooperativos, 100, 134-159.

ITURRIOZ, J. (2013): "Las sociedades cooperativas". En: CAMPUZANO, A. \& PASCUAL, M (Coords.), La empresa: información jurídica, económica y financiera, organización y gestión de personal, Tirant lo Blanch, Valencia, 125-140.

ITURRIOZ, J. \& ISABEL, C. (2010): "La Responsabilidad Social en las sociedades cooperativas: una perspectiva económico financiera" Cuadernos de Estudios Empresariales, 19, 153-173.

ITURRIOZ, J. \& MARTíN, S. (2013). "El tamaño como elemento determinante de la insolvencia en las sociedades cooperativas: estudio a partir de los procesos concursales", REVESCO, Revista de Estudios Cooperativos, 111, 90-107. 
JOVANOVIC, B. (1982): "Selection and the Evolution of Industry", Journal of the Econometric Society, $50,649-670$.

LABATUT, G., POZUELO, J. \& VERES, E.J. (2009): "Modelización temporal de los ratios contables en la detección del fracaso empresarial de la PYME española", Revista Española de Financiación y Contabilidad, 143, 423-447.

LEMMON, M., MA, Y.Y. \& TASHJIAN, E. (2009): "Survival of the fittest? Financial and economic distress and restructuring outcomes in Chapter 11", Third Singapore International Conference on Finance, Singapur.

LINCOLN, M. (1984): "An empirical study of the usefulness of accounting ratios to describe levels of insolvency risk", Journal of Banking and Finance, 8, 321-340.

LIZARRAGA, F. (1997): "Utilidad de la información contable en el proceso de fracaso: análisis del sector industrial de la mediana empresa española", Revista Española de Financiación y Contabilidad, 93, 871-915.

LÓPEZ, J., GANDÍA, J. \& MOLINA, R. (1998): "La suspensión de pagos en las pymes: una aproximación empírica", Revista Española de Financiación y Contabilidad, 94, 71-97.

MANZANEQUE M., BANEGAS R., GARCÍA PÉREZ, D. (2010): "Diferentes procesos de fracaso empresarial. Un análisis dinámico a través de la aplicación de técnicas estadísticas clúster", Revista Europea de Dirección y Economía de la Empresa19, 67-88.

MARÍ, S., MARTÍN, M., SEGUÍ, E. \& MICHAEL, M. (2014): "Análisis cualitativo de los determinantes en la predicción del fracaso empresarial en cooperativas agroalimentarias", ITEA, Información Técnica Económica Agraria: Revista de la Asociación Interprofesional para el Desarrollo Agrario (AIDA), 3, 300-320.

MAROTO, J. (1994): "El equilibrio económico-financiero de la empresa". En: FERNÁNDEZ, A.I (Dir.), Introducción a las Finanzas, Editorial Civitas, Madrid, 113-149.

MARTÍN, S., LEJARRIAGA, G. \& ITURRIOZ, J. (2007): "La naturaleza del capital social como aspecto diferenciador entre las sociedades cooperativas y las sociedades laborales", CIRIEC-España, Revista de Economía Pública, Social y Cooperativa, 58, 59-82.

MARTÍN, M. \& SÁEZ, F.J. (2001): "Políticas públicas de apoyo a la financiación de la pyme: fundamentos, instrumentación y resultados", Papeles de Economía Española, 89/90, 167-186.

MARTIN, D. (1977): "Early warning of bank failure: A logit regression approach", Journal of Banking and Finance, 3, 249-276.

MATA, J. \& PORTUGAL, P. (1994): "Life duration of new firms", The Journal of Industrial Economics, $42,227-245$. 
MATEOS, A. (2008): "Los procesos concursales en sociedades cooperativas. Especificidades en la información económico-financiera”, CIRIEC-España, Revista de Economía Pública, Social y Cooperativa, 60, 209-246.

MELLE, M. (2001): "Características diferenciales de la financiación entre las pyme y las grandes empresas españolas. Asimetrías informativas, restricciones financieras y plazos de endeudamiento", Papeles de Economía Española, 89/90, 140-166.

MÍNGUEZ, J.L. (2006): "Factores explicativos de la insolvencia empresarial: una aplicación a la pequeña y mediana empresa constructora", VI Jornadas sobre Predicción de Insolvencia Empresarial, Carmona (Sevilla).

OHLSON, J.A. (1980): "Financial ratios and the probabilistic prediction of bankruptcy", Journal of Accounting Research, 18, 109-131.

PEÑA, D. (2001): Fundamentos de Estadística, Madrid: Alianza Editorial.

PHILLIPS, B.D. \& KIRCHHOFF, B.A. (1989): "Formation, growth and survival; small firm dynamics in the US economy", Small Business Economics, 1, 65-74.

PLATT, H. \& PLATT, M. (1991): "A note in the use of industry-relative ratios in bankruptcy prediction", Journal of Banking and Finance, 6, 1183-1194.

POZUELO, J., LABATUT, G. \& VERES, E. (2013): "Validez de la información financiera en los procesos de insolvencia. Un estudio de la pequeña empresa española", Cuadernos de Economía y Dirección de la Empresa, 16, 29-40.

POZUELO, J., MARTíNEZ, J. \& CARMONA, P. (2012): "Estudio de la insolvencia empresarial en las cooperativas mediante técnicas multivariantes", Estudios de Economía Aplicada, 3, 1-23.

PREMACHANDRA, I., BHABRA, G. \& SUEYOSHI, T. (2009): "DEA as a tool for bankruptcy assessment: A comparative study with logistic regression technique", European Journal of Operational Research, 193, 412-424.

RINGLE, C.M., WENDE, S. \& BECKER, J.M. (2015): SmartPLS 3, SmartPLS GmbH, http://www.smartpls.com, Boenningstedt.

ROMÁN, I., DE LA TORRE, J.M. \& ZAFRA, J.L. (2001): "Análisis sectorial de la predicción del riesgo de insolvencia: un estudio empírico", XI Congreso AECA: Empresa, Euro y Nueva Economía.

RUBIO, M. (2008): "Análisis del fracaso empresarial en Andalucía. Especial referencia a la edad de la empresa", Cuadernos de Ciencias Económicas y Empresariales, 54, 35-56.

RUIZ-MAYA, L. \& MARTíN- LÓPEZ, F.J. (2005): Fundamentos de Inferencia Estadística, Madrid: Thomson Paraninfo.

SÁEZ, F.J., \& GONZÁLEZ, F.J. (2004): "Las empresas de trabajo asociado en España: regulación, funciones e importancia cuantitativa", Mediterráneo Económico, 6, 133-145. 
SALA, M., FARRÉ, M. \& TORRES, T. (2014): "Un análisis del comportamiento cíclico de las cooperativas y sociedades laborales españolas y su relación con la actividad económica", REVESCO, Revista de Estudios Cooperativos, 115, 7-29.

SINKEY, J. (1975): "A multivariate statistical analysis of the characteristics of problem bank", The Journal of Finance, 30, 21-36.

STONE, M. (1974): "Cross-validatory choice and assessment of statistical predictions", Journal of the Royal Statistical Society: Series B, 36, 111-147

TAFFLER, R. (1983): "The assessment of company solvency and performance using a statistical model: A comparative UK based study", Accounting and Business Research, 52, 295- 307.

WINAKOR, C. \& SMITH, R. (1935): "Changes in Financial Structures of Unsuccessful Industrial Companies", Bureau of Economic Research, 51, University of Illinois Press, Illinois. 